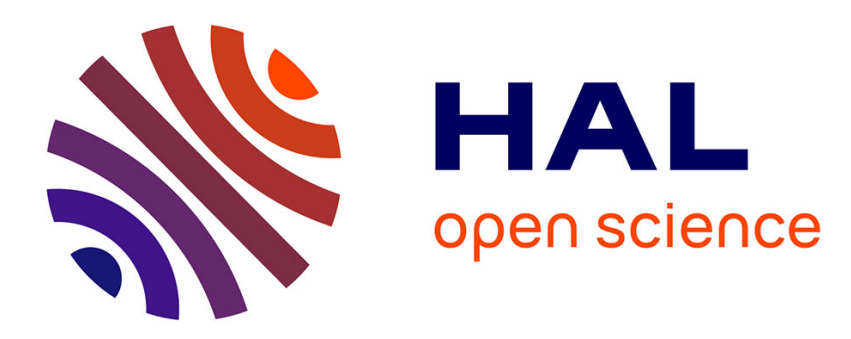

\title{
Latest developments in $(\mathrm{e}, 2 \mathrm{e})$ on solids
}

\author{
E. Weigold
}

\section{To cite this version:}

E. Weigold. Latest developments in (e,2e) on solids. Journal de Physique IV Proceedings, 1993, 03 (C6), pp.C6-187-C6-196. 10.1051/jp4:1993618 . jpa-00251703

\section{HAL Id: jpa-00251703 https://hal.science/jpa-00251703}

Submitted on 1 Jan 1993

HAL is a multi-disciplinary open access archive for the deposit and dissemination of scientific research documents, whether they are published or not. The documents may come from teaching and research institutions in France or abroad, or from public or private research centers.
L'archive ouverte pluridisciplinaire HAL, est destinée au dépôt et à la diffusion de documents scientifiques de niveau recherche, publiés ou non, émanant des établissements d'enseignement et de recherche français ou étrangers, des laboratoires publics ou privés. 


\title{
Latest developments in (e,2e) on solids
}

\author{
E. WEIGOLD
}

Research School of Physical Sciences and Engineering, Australian National University, Canberra 0200, Australia

\begin{abstract}
Recent progress in the application of the $(e, 2 e)$ technique for elucidating the electronic structure of condensed matter is discussed. Particular emphasis is given to the description of a new third generation electron momentum spectrometer, which operates with an incident beam energy of 20-30 keV, uses asymmetric non-coplanar scattering kinematics with scattered electrons emerging at polar angles $\theta_{s}=14^{\circ}$, and the ejected electrons emitted at $\phi_{e}=76^{\circ}$. A range of scattered and ejected electron energies $(20 \mathrm{eV})$ and azimuthal angles $\left(-18^{\circ} \leq \phi_{s} \leq 18^{\circ},-7^{\circ} \leq \phi_{e}-\pi \leq 7^{\circ}\right)$ are detected in parallel by means of two-dimensional position sensitive detectors. Software determines the energies and angles of the detected electrons, corrects for flight times through the analysers, and determines the separation energy and momentum for each event. With a $5 \mathrm{~nm}$ amorphous carbon membrane, the true signal rate is $6 \mathrm{~Hz}$ and the signal to background ratio is 1 with an incident current of $100 \mathrm{nA}$. Without monocromation of the incident beam the energy resolution is $1 \mathrm{eV}$ and the momentum resolution is $0.15 \mathrm{au}$. The spectral momentum densities obtained for evaporated amorphous carbon are compared with theoretical results.
\end{abstract}

\section{INTRODUCTION}

The first (e,2e) measurement with emphasis on structure determination was a high energy experiment on a solid thin film of carbon [1], preceding the first such measurements on a gas target [2] by a few months. However, for the solid target the energy resolution was over $100 \mathrm{eV}$ and the momentum resolution was between 1.5 and $2 a u$. With such poor energy and momentum resolution no meaningful comparison with theory could be made. On the other hand, for the argon gas target measurements [2] the energy resolution was only a few $\mathrm{eV}$ and the momentum resolution approximately $0.15 \mathrm{au}$, and this work showed for the first time the ability of EMS to map momentum densities for target electrons as well as for determining correlation effects in the valence shell. Since then the technique of EMS has developed into a powerful tool for the study of the electronic structure of gas phase atoms and molecules and their ions [3].

The application of EMS to condensed matter targets should reveal a host of new information on their electronic structure. Consider an electron in a solid with binding energy $\varepsilon$ (relative to the Fermi energy) and real momentum q. In crystalline solids the binding energy and momentum 
are correlated through the dispersion relation $\varepsilon=\varepsilon(\mathbf{k})$ where $\mathbf{k}$ is in the first Brillouin zone and $\mathbf{q}=\mathbf{k}+\mathbf{G}$ where $\mathbf{G}$ is the reciprocal lattice vector. The distinctive feature of EMS is that it allows a direct measurement of $\varepsilon(\mathbf{k})$ and $\mathbf{q}$ through measurement of the incident and outgoing momenta $\mathbf{k}_{i}, \mathbf{k}_{s}$ and $\mathbf{k}_{e}$ [3], but in addition it obtains directly the spectral electron momentum density through the cross section

$$
\left[\frac{d^{5} \sigma}{d \Omega_{s} d \Omega_{e} d E_{s}}\right]=C \rho_{\mathbf{k}, \alpha}(\varepsilon, \mathbf{q})
$$

where $\rho_{\mathbf{k}, \alpha}(\varepsilon, \mathbf{q})$ is the spectral momentum density for band $\alpha$ and $\mathrm{C}$ is a kinematical factor which is essentially a constant under normal EMS conditions. For crystalline solids, in the independent electron approximation,

$$
\rho_{\mathbf{k}, \alpha}(\varepsilon, \mathbf{q})=\delta\left(\varepsilon-\varepsilon_{\mathbf{k} \alpha}(\mathbf{q})\right)\left|\phi_{\mathrm{k} \alpha}(\mathbf{q})\right|^{2},
$$

where $\left|\phi_{\mathrm{k} \alpha}(q)\right|$ is the one-electron momentum density. For an amorphous target consisting of randomly oriented micro-crystals, the (e,2e) cross section (eq. (1)) is proportional to the spherically averaged spectral momentum density

$$
\left\langle\rho_{\mathbf{k}, \alpha}(\varepsilon, \mathbf{q})\right\rangle=\frac{1}{4 \pi} \int d \hat{q} \rho_{\mathbf{k}, \alpha}(\varepsilon, \mathbf{q})
$$

EMS is the only technique that can measure directly the momentum densities of individual bands as well as the dispersion curves of electrons in solids. However, EMS in solids has progressed much more slowly than in gases due to the difficulty of studying high density targets in transmission. High energy electrons are required to maximise the mean free path in a solid and to minimize the strong multiple scattering effects. But as the incident energy $E_{i}$ is increased the (e,2e) cross section decreases, and it becomes increasingly difficult to obtain good momentum and energy resolution. Twelve years after the initial measurements on carbon [1], Ritter et al [4] were able to resolve for the first time some valence structure in a solid target (again amorphous carbon) with $25 \mathrm{keV}$ incident electrons and $6 \mathrm{eV}$ energy resolution. Since then Hayes et al $[5,6]$ and the Flinders group $[7,8]$ improved these measurements with energy (momentum) resolutions of $4.5 \mathrm{eV}$ $\left(0.6 a u^{-1}\right)$ and $1.5 \mathrm{eV}\left(0.15 a u^{-1}\right)$ respectively. The work of Hayes et al $[6]$ and of Lower et al $[7,8]$ utilized one-dimensional multiparameter $(e, 2 e)$ spectrometers which could measure over a range of binding energies simultaneously, thus significantly improving the rate of acquiring data. More recently Storer et al [9] have made measurements at $20 \mathrm{keV}$ incident energy on various forms of amorphous carbon with a new type of coincidence spectrometer, one which uses multidetection of both angles and energies of the outgoing electrons. This spectrometer allows for the first time the measurement of a range of both binding energies and recoil momenta in parallel, leading to a greatly enhanced sensitivity. It makes it possible to apply EMS to the detailed investigation of the electronic structure of condensed matter.

In the remaining sections this new spectrometer is discussed briefly and its sensitivity indicated with some measured examples using amorphous carbon as the target material. 


\section{APPARATUS}

\subsection{Scattering Geometry}

The spectrometer employs asymmetric Bethe ridge kinematics for which the high energy scattered electron $\left(k_{s}>>k_{e}\right)$ is emitted at an angle given by

$$
\cos \theta_{s}=\left(k_{s}^{2}+\varepsilon\right) / k_{i} k_{s}
$$

We use the normal convention of naming the faster and slower emitted electrons respectively the scattered and ejected electrons. The momentum transfer $K=\mathbf{k}_{i}-\mathbf{k}_{\mathbf{s}}$ is much smaller in this kinematics than in the symmetric kinematics normally employed in EMS, thus the cross section which goes as $K^{-4}$ is much larger at a given incident energy $E_{i} . K$ must, however, be kept large to ensure close electron-electron, i.e. binary collisions. In the new Flinders spectrometer with $E_{i}=20 \mathrm{keV}, \theta_{s}=14^{\circ}, E_{s}=18.8 \mathrm{keV}, E_{e}=1.2 \mathrm{keV}, \theta_{e}=76^{\circ}$ for $q=0$ and $K=9.4 a u^{-1}$.

The asymmetric geometry has the additional advantage of making it significantly easier to obtain good energy resolution. Only one high voltage power supply is needed. It is connected to both the electron gun and the scattered electron analyser. Thus any effects of drifts and ripples in this supply are cancelled out, influencing $E_{i}$ and $E_{\text {s }}$ equally. High stability lower voltage supplies $(1.2 \mathrm{keV})$ for the ejected electron spectrometer $\left(E_{e}\right)$ and the equivalent offset supply for incident energy determination and the programmable separation (or binding) energy supply (fig.1) ensure long term stability and accuracy in determining the target electron binding energy

$$
\varepsilon=E_{i}-E_{s}-E_{e}
$$

and momentum

$$
q=k_{e}+k_{s}-k_{i}
$$

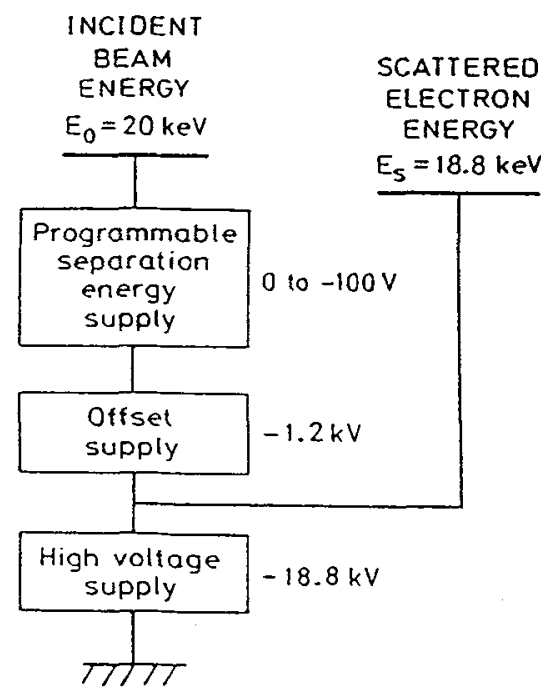

EJECTEO

ELECTRON

ENERGY

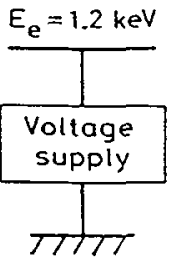

Fig.1: The high voltage supply configuration defining the incident and emitted electron energies. 
A schematic view of the apparatus is shown in fig.2.

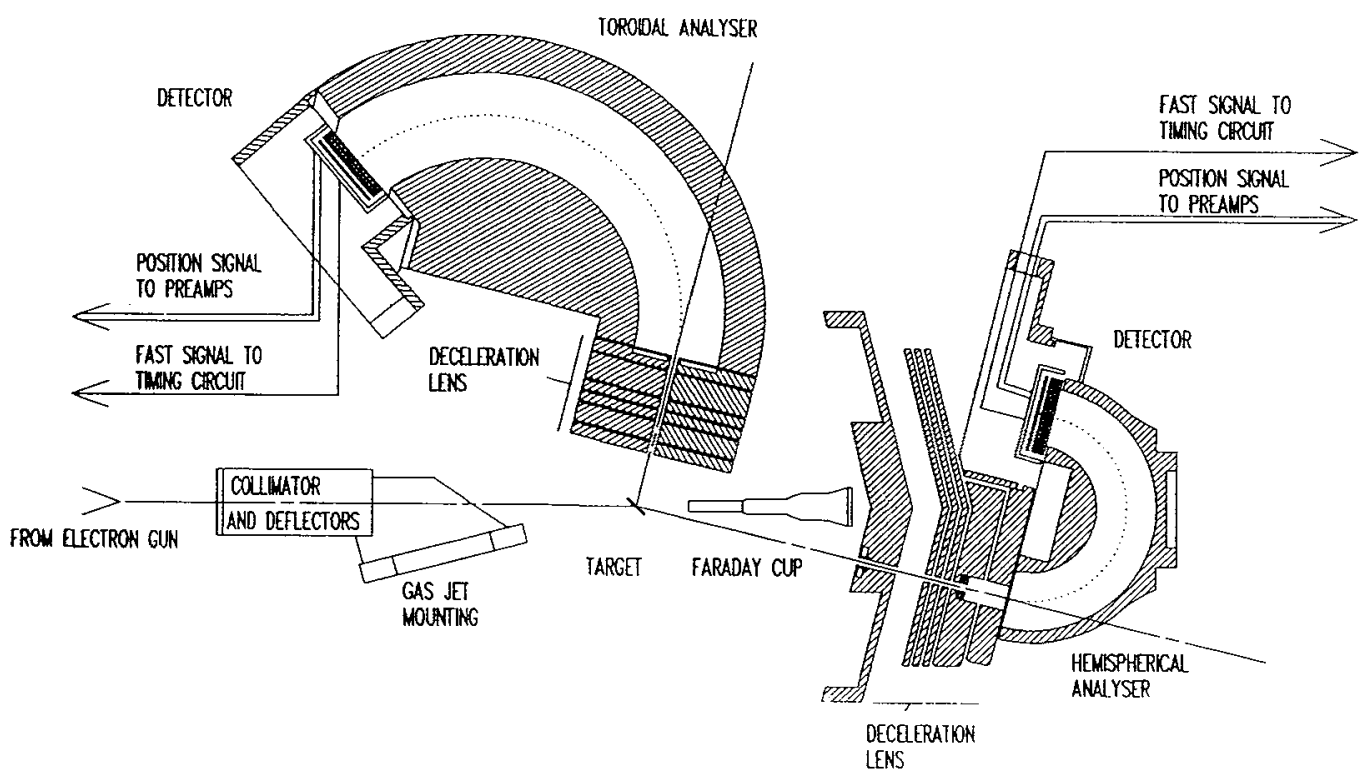

Fig.2: Schematic view of the spectrometer showing incident beam direction and hemispherical and toroidal analysers for determining the energies and azimuthal angles of the fast forward scattered and the slower (ejected) electrons.

\subsection{Electron Optics and Analysers}

The scattered and ejected electron analysers are of hemispherical and toroidal electrostatic deflector design respectively. The decelerating lenses and the deflectors were designed to have axial or near axial symmetry with acceptance cones of $14^{\circ}$ and $76^{\circ}$ respectively. Each analyser detects electrons over a pass band of about $20 \%$ of their mean pass energy normally set at $100 \mathrm{eV}$ and each analyzer accepts a range of azimuthal angles. This allows the spectrometer to simultaneously observe a range of binding energies and a large range of target electron momenta perpendicular to the incident beam $\left(\mathbf{k}_{i}\right)$, up to $4 a u$ with $\phi_{s} \varepsilon\left[-18^{\circ}, 18^{\circ}\right]$ and $\left(\phi_{e}-\pi\right) \varepsilon\left[-6^{\circ}, 6^{\circ}\right]$.

The scattered and ejected electrons transmitted by the analysers within the predetermined energy bands and azimuthal angle ranges (see above) are detected by an array of $40 \mathrm{~mm}$ active diameter multichannel plates followed by a two dimentional resistive anode plate (RA). The position determining pulses are taken from the four corners of the RA and the fast timing pulse from the back of the last multichannel plate. Each analyser is calibrated by mounting an aperture plate in front of the entrance slit to the retarding lens stack with holes drilled at known angles, and by using electrons of known energy elastically scattered by a thin (5-8nm) carbon target to cover the acceptance energy of the analyser. Fig. 3 shows such a calibration run for $18.8 \mathrm{keV}$ electrons for the scattered electron hemispherical deflector system. The peaks correspond to particular energies and angles, with the energy being stepped by $2 \mathrm{eV}$ (radial direction) and the azimuthal angle by $3^{\circ}$ (circumference direction). This data, obtained with a non-energy-selected thermionic tungston 
filament source (i.e. $\Delta E_{i} \sim 0.5 \mathrm{eV}$ ) shows that the angular and energy resolution of the spectrometer are excellent. A similar performance is obtained with the toroidal analyser. A centroid for the $(x, y)$ position of each peak is automatically located by peak recognition software and tabulated along with the known angle and energy of the peak. A polynomial fitting procedure [10] is subsequently used to define the transformation between the $(x, y)$ arrival position coordinate as determined by charge division, and the energy and angle of each detected electron.
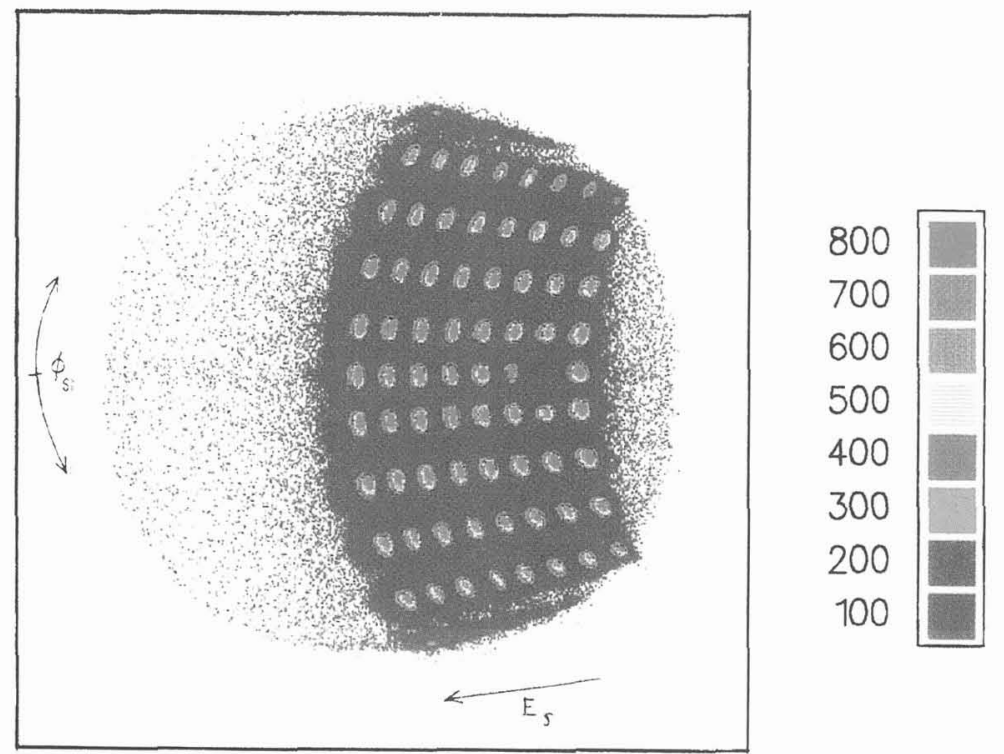

Fig.3: Calibration run of the hemispherical analyser obtained by elastic scattering from a thin film of amorphous carbon through $0.16 \mathrm{~mm}$ diameter angle calibration apertures at $\phi_{s}=0, \pm 3^{\circ} \pm$ $7^{\circ}, \pm 11^{0} \pm 15^{\circ}$, and at incident energies ranging from 18.870 to $18.894 \mathrm{keV}$ in $2 \mathrm{eV}$ steps.

\subsection{Electronics}

The total charge collected by the RA diffuses to the four corners of the anode and after amplification is fed to the input of four of nine gated parallel-input ADC's (fig.4). From the knowledge of the charge collected at the four corners and the total charge, the position of arrival can easily be determined [10]. The outputs from the other resistive anode are similarly fed into four of the remaining ADC's and the ninth $\mathrm{ADC}$ is used for the timing information provided by the TAC, which measures the time difference of arrival of electrons in the detectors. A gate signal from the TAC opens the gates whenever two electrons were detected within 100nsec of each other.

The multiparameter acquisition system has a tenth parallel data channel, the tenth being used to store the separation energy setting. A dual channel buffer memory system allows data conversion and subsequent storage in one $8 \mathrm{k}$ buffer, while the second $8 \mathrm{k}$ buffer is being read by the online computer. 
The base of the observed coincidence peak from the TAC is about 20ns wide. However, since the arrival position of the electron is known, its trajectory and time of flight through the deflector can be calculated. Such correction by software for time-of-flight through the analysers reduces the coincidence width to about 5ns with FWHM of $2 \mathrm{~ns}$, therefore considerably enhancing the signal to noise ratio.

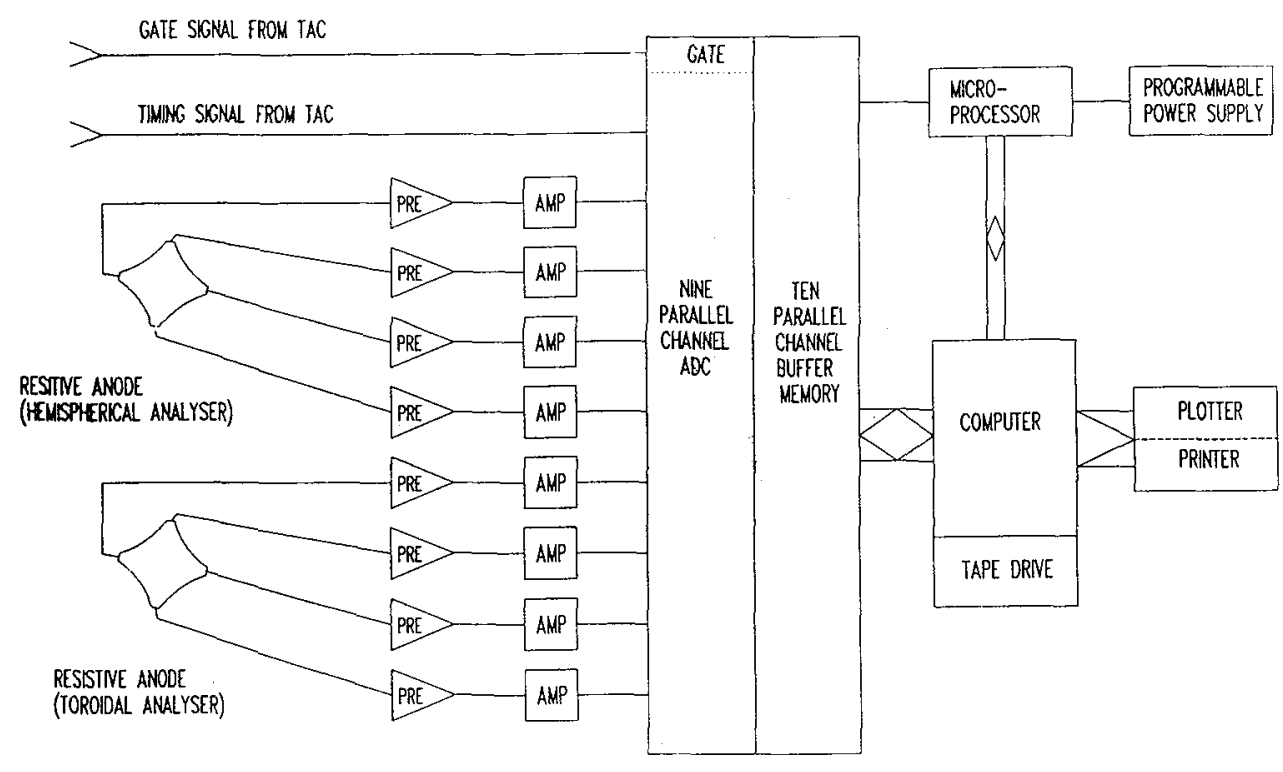

Fig.4: Schematic of "slow" pulse circuit, showing the input to the multiparameter acquisition system (MAS) and computer. Not shown is the fast timing and MAS gating circuitry.

The binding energy and momentum are also calculated for each event and stored. The separation energy is swept through a large range by stepping the programmable separation energy power supply through the appropriate range, similar to the binning mode described by Cook et al [11]. The true coincidences are determined by subtraction of accidental coincidences in the normal way [3]. The background accidental coincidences are also used to determine the instrumental response function.

\section{RESULTS}

The true coincidence count rate and signal to background ratio obtained with a $5 \mathrm{~nm}$ thick amorphous carbon target and an incident current of $150 \mathrm{nA}$ are typically $6 \mathrm{~Hz}$ and 1 respectively. A set of data that required about 100 hours of data acquisition is shown in fig.5(a). As well as showing the spectral momentum density of evaporated amorphous carbon, it includes contributions due to multiple scattering in the target. The dominant inelastic multiple scattering loss in a-C is due to plasmon excitation, which gives rise to a broad peak at $25 \mathrm{eV}$ energy loss. This is measured separately for incident and ejected-electrons by using incident electron beams of the appropriate energies and measuring the elastic and inelastic non coincidence signal. Corrections for multiple scattering are straight forward [12], and fig.5(b) shows the resulting spectral momentum density contour map with multiple scattering removed. 




$$
\text { (a) }
$$
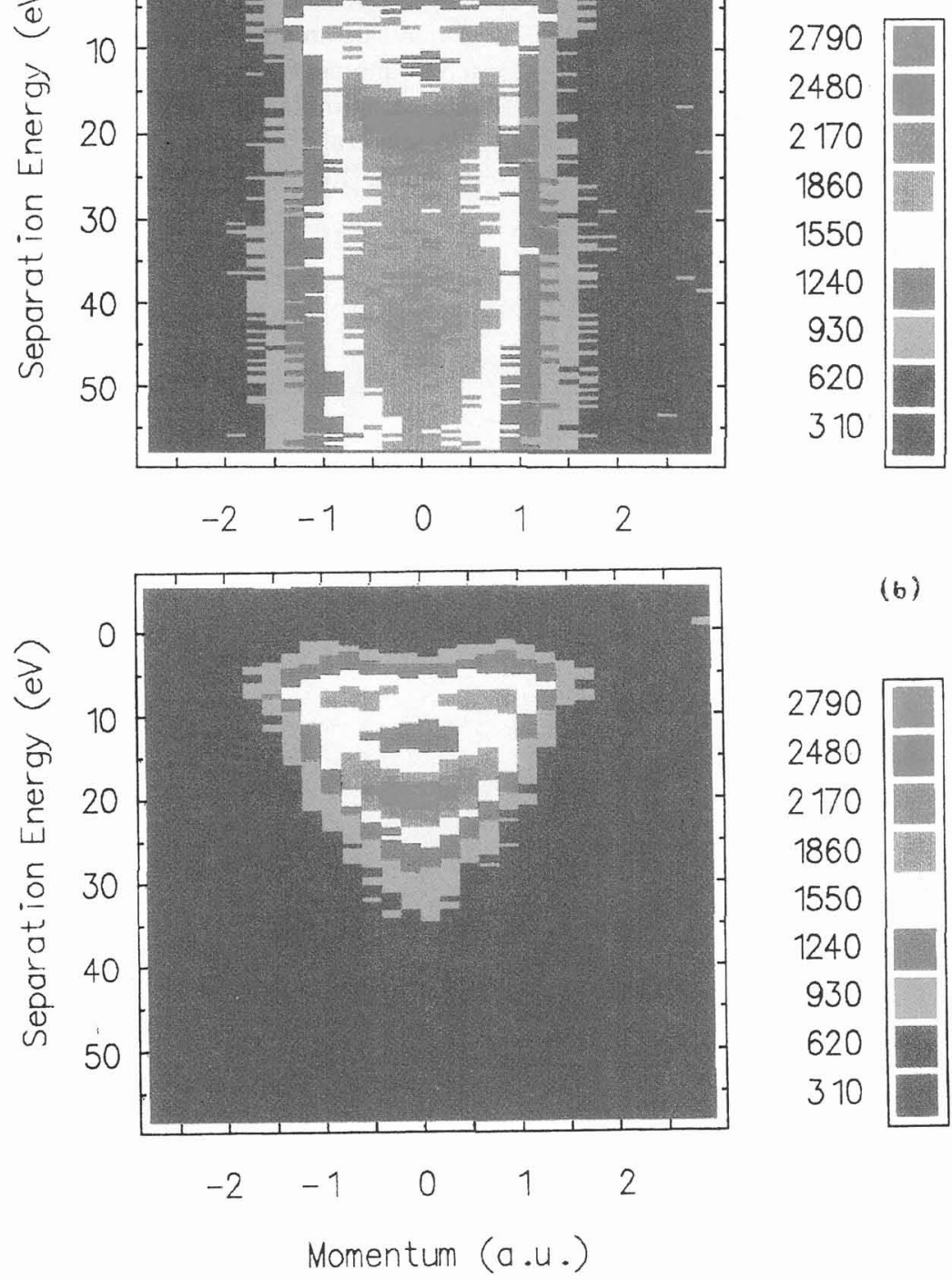

(b)

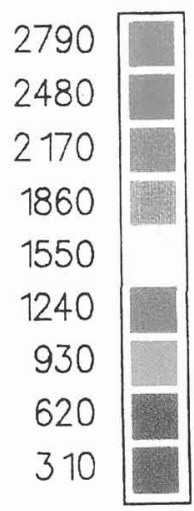

Fig.5: (a) Raw coincidence data for $(e, 2 e)$ on a-C showing the computer graphics display in one of its modes, i.e. coincident count rate as a function of separation energy and momentum. The coincidence counts per pixel are colour coded as indicated. (b) The spectral momentum density for a-C obtained by correcting the raw data in 5(a) for multiple scattering effects. 


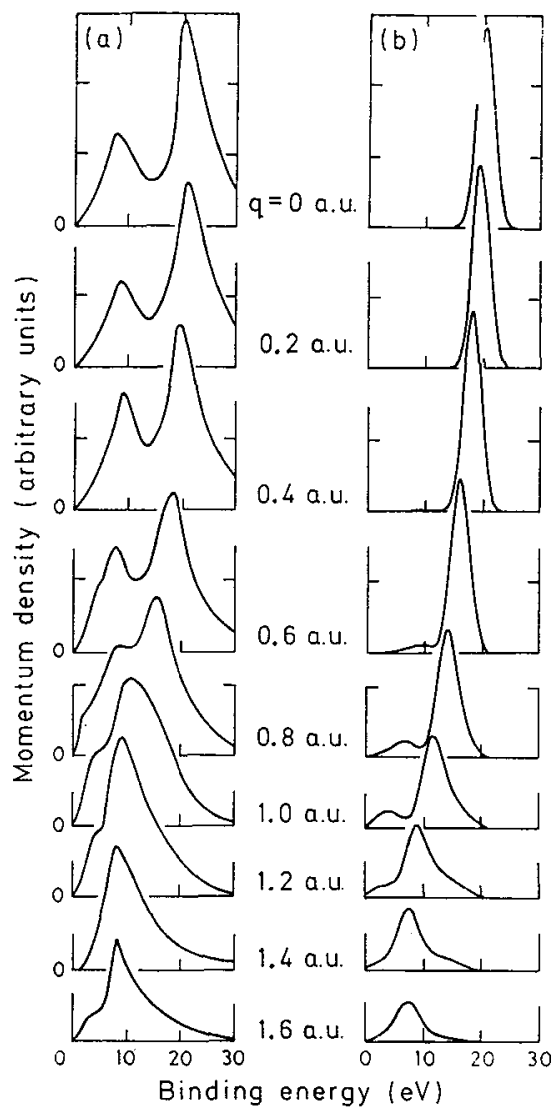

Integral Intensity of the Peaks

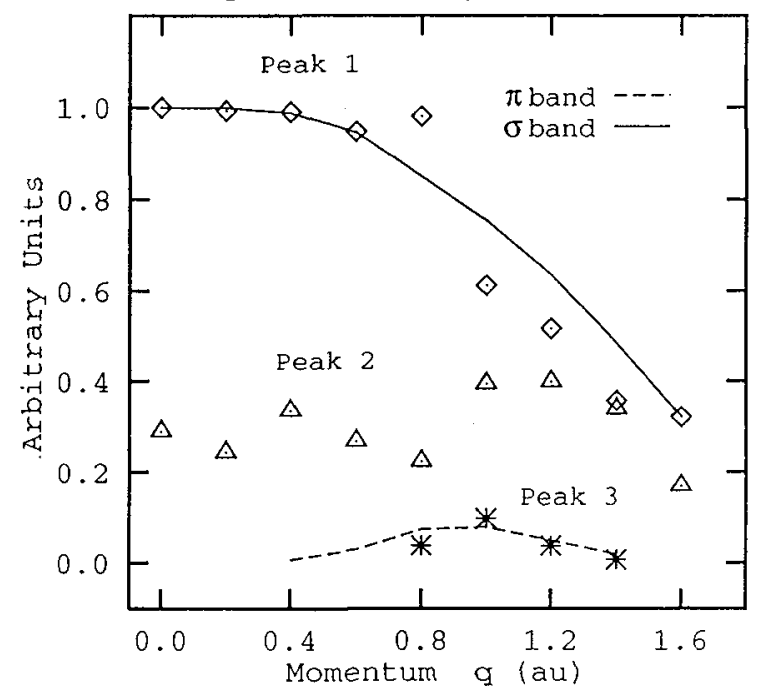

Fig.6: Spectral momentum densities for amorphous carbon (binding energy spectra) for different momenta (a) measured [13] and (b) numerical simulation by spherically averaging the spectral momentum density of crystalline graphite [13].
Fig.7: Dispersion with momenta of the three features shown in fig 6(a). The diamonds are from the inner valence band, triangles from the lower binding energy peak and asterisks from the low energy shoulder observed at $q \geq 0.6$. The solid and broken lines are the LMTO calculation for the $\sigma$ and $\pi$ bands, respectively [13]. 
Several features are immediately obvious. The first is that the highest density is at $q \sim 0$ and $\varepsilon \sim 20 \mathrm{eV}$. This band shows considerable dispersion and the density decreases monotonically as $q$ increases. The second feature is the band at $\varepsilon \sim 7 \mathrm{eV}$, which has a maximum at $q \sim$ $0.75 a u$ and shows little or no dispersion. These two features have previously in the low resolution measurements (e.g. ref [4]) been associated with the $\sigma$ and $\pi$ bands of graphite, evaporated amorphous carbon being considered graphitic in nature, perhaps consisting of randomly oriented microcrystals of graphite.

Kheifets [13] has carried out a linear muffin-tin orbital calculation for the electronic structure of graphite, and then spherically averaged in momentum space the resulting dispersion curves and momentum densities. His result is shown in fig.6(b) together with some data obtained using a symmetric coplanar spatrometer [13,7]. The agreement between the calculated innervalence $\sigma$ band and the observed band $\varepsilon=20 \mathrm{eV}$ at $q=0$ is excellent. However, the calculations show that the $\pi$ band should have a very small density peaked at about $q \sim 1 a u$ with vanishing density at small $q$. The measured density for the $7 \mathrm{eV}$ band is much higher at all $q$.

Close examination of the data in fig 5 and 6(a) shows the presence of a shoulder at $\varepsilon \sim 5 \mathrm{eV}$ in the $q$ range 0.6-1.6. An attempt at deconvoluting this feature and the other two main structures, leads to the band momentum densities shown in fig.7. They are also compared with those calculated by Kheifets et al, and good agreement is seen for the inner $\sigma$ band and the outermost feature, which corresponds to the $\pi$ band. However, the strong feature at $\varepsilon \sim 7 \mathrm{eV}$ is unexplained. Diamond like amorphous carbon should also have no such feature. Auger spectroscopy of the films eliminated possible contaminants such as oxygen. Hydrogen contamination does occur, but only at the few percent level. It is quite possible that this feature could be due to various molecular forms of carbon, which may be present in the film. The origin of this feature is still an open question which is currently being investigated.

\section{CONCLUSIONS}

Clearly the technique of ESM can reveal rich new information on the electronic structure of solids. It can measure the full spectral momentum densities of suitable targets. The energy resolution can be easily improved by adding a monochrometer, since currents of only a hundred nanoamperes or so are required. The main limitations at present are the difficulties involved in obtaining thin target materials. However, these can and are being overcome, and we can look forward to measurements on crystals, semiconductors, superconductors, and surfaces. In the case of surfaces, grazing incidence geometry may be more appropriate. In the case of superconductors it would be fascinating to map the wavefunctions both above and below the critical temperatures.

\section{ACKNOWLEDGEMENTS}

I am grateful to Peter Storer, Robert Caprari, Steve Clark, Ian McCarthy, Bruce Stevens, Ian Smart, Mark Ellis and all the others who helped in this work. I am also grateful to the ARC and the ANU for financial support. 


\section{REFERENCES}

[1] Camilloni R., Ciardini-Guidoni A., Tirebelli R. and Stefani G., Phy.Rev.Lett. 29 (1972) 618

[2] Weigold E., Hood S.T., and Teubner P.T.O., Phys.Rev.Lett. 30 (1973) 475

[3] McCarthy I.E. and Weigold E.,Rep.Prog.Phys.51 (1988) 299, ibid 54 (1991) 789

[4] Ritter A.L., Dennison J.R. and Jones R.,Phys.Rev.Lett. 53 (1984) 2054

[5] Hayes P., Bennett M.A., Flexman J. and Williams J.F., Phys.Rev.B. 38 (1988) 13371

[6] Hayes P., Williams J.R. and Flexman J., Phys.Rev.B.43 (1991) 1928

[7] Lower J., Bharathi S.M., Chen Y., Nygaard K.J. and Weigold E., Surf.Sci. 251/252 (1991) 213

[8] Nygaard K., Chen Y., Lower J., Storer P. and Weigold E. Microsc.Microanal Microstruct. 2 (1991) 377

[9] Storer P., Caprari R.S., Clark S.A.C. and Weigold E Rev.Sci.Inst. (submitted)

[10] Caprari R.S., Computers in Physics 7 (1993) 336

[11] Cook J.P.D., McCarthy I.E., Stelbovics A.T. and Weigold E., J.Phys.B. 17 (1984) 2339.

[12] Storer P. (1993) PhD Thesis, Flinders University

[13] Kheifets A.S, Lower J., Nygaard K.J., Utteridge S., Vos M., Weigold E., and Ritter A.L. (1993) to be published. 